\title{
Quality of life and owner attitude to dog overweight and obesity in Thailand and the Netherlands
}

\author{
Nienke Endenburg ${ }^{1 *}$ (D), Sirikul Soontararak ${ }^{2,3,4}$, Chalermpol Charoensuk ${ }^{3}$ and Hein A. van Lith 2,5
}

\begin{abstract}
Background: This study investigated whether the body condition score (BCS) and/or culture influences the quality of life (QoL) of dogs, as evaluated by the owner, and whether the BCS is influenced by feeding and exercise and its owner's culture. To this end, a questionnaire was administered to 355 selected dog owners (Thai and Dutch). Their dogs had a BCS of 3 (normal weight), 4 (overweight) or 5 (obese) but no other physical problems. Instead of using Likert scales, continuous scales were used. Further, data for the questionnaire items were transformed using an integrated z-score methodology.

Results: The magnitude of factor loadings was similar to that reported in a previous study, indicating that the questionnaire is not culture specific. QoL scores for general sickness were significantly higher (worse) in dogs with a higher BCS. Thus even though the dogs were apparently healthy, the BCS influenced the perceived QoL of the dog. Immobility was seen more often in dogs with a higher (poorer) BCS than in dogs with a lower (better) BCS; however, there was no clear relationship between immobility and total activity. The higher the BCS, the less owners felt in control of feeding and exercise. The BCS was higher in the dogs of owners who did not like to exercise. The Thai dogs showed more separation-related behaviour problems when their owner left home than did the Dutch dogs.

Conclusions: The QoL of overweight and obese dogs is mainly influenced by the dog's physical status. The owners of dogs with a high BCS have less perceived control over feeding and exercise. Our findings indicate that owner attitudes and beliefs essentially cause obesity as a result of a lack of knowledge and perceived control.
\end{abstract}

Keywords: Body condition score (BCS), Cultural influence, Dogs, Exercise, Feeding, Obesity, Overweight, Quality of Life (QoL)

\section{Background}

Overweight is an increasingly common problem in humans and their pets $[1,2]$. New standards have been set for dogs, with obesity being defined as a weight greater than $15 \%$ of ideal [3]; standards that have consequences for health and wellbeing $[4,5]$. Up to $40 \%$ of dogs in developed countries are overweight [6]. The most common cause of overweight is an energy intake exceeding energy expenditure. Several risk factors for overweight have been identified, such as breed/genetic background [7], neuter status [8], orthopaedic diseases (and thus decreased activity) [9], type of diet [10], change of lifestyle [11], body

\footnotetext{
* Correspondence: N.Endenburg@uu.nl

${ }^{1}$ Division Human-Animal Relations, Department of Animals in Science and Society, and Animal Behaviour Clinic, Faculty of Veterinary Medicine, Utrecht University, Yalelaan 2, PO Box 80166, 3508, TD, Utrecht, The Netherlands Full list of author information is available at the end of the article
}

condition score (BCS) of the dog as judged by its owner [10], and owner socioeconomic status $[9,11]$.

Overweight and obesity are a growing problem in distinct companion dog populations and can cause discomfort and disease, and/or reducing the quality of life (QoL) of affected dogs [12]. Obesity can promote the development of a number of diseases that reduce the animal's QoL and life span [13]. The general concept of QoL takes into account the physical, mental, and social needs of the individual, and meeting these needs is considered to reflect a positive status. Therefore, QoL assessment can be used to assess improvements in conditions that adversely affect QoL, such as prolonged discomfort and disease.

In humans, the perception of health and illness, and hence QoL, are culturally determined [14] and thereby

(c) The Author(s). 2018 Open Access This article is distributed under the terms of the Creative Commons Attribution 4.0 International License (http://creativecommons.org/licenses/by/4.0/), which permits unrestricted use, distribution, and reproduction in any medium, provided you give appropriate credit to the original author(s) and the source, provide a link to the Creative Commons license, and indicate if changes were made. The Creative Commons Public Domain Dedication waiver (http://creativecommons.org/publicdomain/zero/1.0/) applies to the data made available in this article, unless otherwise stated. 
influence the relative importance of factors such as affection, satisfaction, and acceptance, factors that are in turn influenced by lifestyle, past experience, and social consensus [15]. A person's beliefs and attitudes to overweight, obesity, eating behaviour, and exercise are also culturally determined [16]. For example, many cultures believe that human overweight reflects wealth, prosperity, and a high status $[16,17]$. However, it is not clear whether cultural beliefs influence how owners perceive the QoL of their pets. Apart from cultural belief influences, climate probably also influences the QoL of pets (dogs), because an owner is less likely to walk or run with their dog in hot weather [18].

The dog-owner relationship may be a source of bias in owner reports of their dog's symptoms, which complicates the work of veterinarians [12]. There is evidence that the beliefs of the owners of normal-weight dogs are different from those of the owners of obese dogs. Kienzle et al. [10] found that the owners of obese dogs were more likely to believe that exercise and balanced dog nutrition were less important than were the owners of normal-weight dogs. Although factors such as older age, gender, breed, and neutering increase the risk of obesity, the disorder is ultimately caused by inappropriate feeding and exercise given by the owners [19]. Bland et al. [20] concluded that obesity in dogs is affected by the interrelationship between food management, exercise, and social factors such as the belief of owners that obesity is not a health problem. Further, it has been found that the extent to which dogs are overweight is related to the body mass index of their owners [21]. Sandøe et al. [22] states that: "We cannot hope to understand feline and canine obesity without also knowing something about human obesity, the social status of owners, and the relationships that humans actually have with their dogs and cats". Obesity in pets and humans is thus a One Health issue [22, 23].

Rohlf et al. [6] designed a questionnaire to determine the extent to which owners have perceived control over the feeding and exercise of their pets. The questionnaire is based on the theory of planned behaviour (TPB) [24] which holds that many behaviours, including behaviours towards animals [25], are predicted primarily by intentions, which in turn are determined by behavioural attitudes, subjective norms, and perceived behaviour control. In humans, TPB explains healthy eating and exercise behaviours [26].

How owners feed and exercise their dogs is probably culturally determined, reflecting a composite of subjective beliefs, religion, normative beliefs, and attitudes [16], although there has been relatively little research in this field. In the Netherlands, with its Judeo-Christian tradition [27], food, feeding, and consumptive practices are important constructs of self-identity. In contrast, in Buddhism, food is a component of positive karma, essential to a being's happiness [28].

Although the assessment of QoL in animals is controversial, it can be measured with well-designed, reliable, and valid questionnaires [12, 29, 30]. Assessment of a dog's QoL can make it possible to assess the impact of discomfort and disease [31]. Obesity may diminish a dog's QoL if it causes physical impairments and disabilities.

The aims of this study were to investigate whether BCS and/or culture (the Netherlands and Thailand) affect the owner-reported QoL of dogs and whether the BCS is influenced by owner attitudes regarding feeding and exercise or the owner's culture.

\section{Methods}

\section{Data collection and questionnaire}

Data were collected at Kasetsart University Veterinary Teaching Hospital (KU-VTH), Thailand, and at veterinary practices and dog grooming parlours in the Netherlands. When the dogs were brought to the KU-VTH or Dutch veterinary practices (such as for vaccination, annual health check-up, spaying/neutering, blood donation, etc.), or to a grooming parlour, their owners were given a questionnaire (see Additional file 1: Appendix) to complete. The owners were informed that this was a study about the quality of life of dogs. The owners were given the questionnaire by the researcher and had to fill in the questionnaire on their own. To prevent that social desirable answers were given, in the introduction letter to the questionnaire it was stated that confidentiality and data protection are promised [32]. The veterinary technician or veterinary student were asked to select dogs that were normal weight (BCS 3), overweight (BCS 4) or obese (BCS 5), but otherwise healthy (Thailand $n=200$, the Netherlands $n=155$ ). The veterinary technician or veterinary student were supervised by a veterinarian who was also available for consultation. The veterinarian/veterinary technician/veterinary student have been trained on body condition scoring. In Thailand the dogs that were used for this investigation, came to the Veterinary Teaching Hospital of Kasetsart in Bangkok. In the Netherlands the dogs came from private practices or grooming parlours and the veterinarians or veterinary students who assessed the animals were all educated at the University Clinic for Companion Animal Health in Utrecht, the Netherlands. Veterinarians in both countries were informed about the inclusion criteria, and selected the dogs accordingly. In both countries, pre-selection of dogs was done by trained veterinary technicians or veterinary students, before the veterinarian selected the dogs. To determine the BCS, the BCS chart from Hill's Pet Nutrition (5 scales silhouette system; http://lasvegaspetweightloss.com/hills-metabolic-diet-program/) was used and the BCS was noted at the beginning of the questionnaire. Scores on this scale determined by different operators have been shown to correlate well, although a degree of expertise is required; which makes the scoring instrument less suitable for owners $[2,8]$.

The questionnaire contained four sections: one about demographic variables, one about the dog's food and 
exercise, one about the dog's QoL (based on the 91-item questionnaire of Schneider et al. [12]; 39 items selected), and one about owner attitudes (based on the 89-item questionnaire of Rohlf et al. [6]; 42 items selected). The original questionnaire of Schneider et al. [12] has been validated, but that of Rohlf et al. [6] has not. Answers were given on a continuous rating scale (visual analogue scale, Fig. 1). The questionnaire was prepared in English and then translated into Thai and Dutch. Although Chen \& Boore [33] advise bilingual translators, the Thai translation was made by five Thai native speakers who were selected from different fields of expertise, such as veterinarians, veterinary technicians, owners graduated in engineering and science, but none of these people were bilingual - they had learned English at school and their study at the university; they were fluent in English. The questionnaire was pre-tested among 11 volunteers in Thailand, as advised by Schellingerhout et al. [34] to increase the validity of the translated questionnaire. It took them about 15 minutes to complete. After adjustment, the questionnaire was distributed among dog owners coming to the KU-VTH. The questionnaire was translated from English into Dutch by the first author together with an English teacher (Dutch native speaker) and three veterinary students. None of these translators were bilingual, but they were fluent in English. The Dutch questionnaire was also pre-tested among $10 \mathrm{dog}$ owners with different educational backgrounds; it took them about 15 minutes to complete. In the pre-testing period, the respondents were asked if they understood the questions. Both the Thai and Dutch questionnaires were in general considered clear so that only minor changes in wording were made. Five questions from part 3 ('My dog has trouble going up and/or down stairs,' 'My dog has difficulty getting in and/or out cars', 'It is easy to train my dog', 'My dog will perform tricks', and 'My dog responds to verbal correction when he/she misbehaves') were left out because they were not specific and could not be used to assess QoL in an overweight and obese population.

\section{Processing of the questionnaires and z-score calculation}

The questionnaires of Rohlf et al. [6] and Scheider et al. [12] used 7-point and 5-point Likert-type scales, respectively. However, Likert-type scales yield ordinal data and thus non-parametric statistical tests should be used, but in general non-parametric tests are less powerful than parametric tests and are also restricted in their application. This implies that parametric compared to non-parametric tests are often inferior. A continuous rating scale makes it possible to use robust parametric statistics and results obtained are less affected by noise. For this reason, the items in the third and fourth parts of the questionnaire were scored on a $10-\mathrm{cm}$ visual analogue scale, going from 0 ('strongly disagree,' 'extremely unlikely', or 'no control at all') to 10 ('strongly agree,' 'extremely likely', or 'complete control') (Fig. 1). Use of a continuous scale made it possible to calculate means and standard deviations (SDs) for the items, so that data could be reduced by creating summary/composite variables.

To obtain comprehensive and integrated summary variables, the data for the questionnaire items were transformed using a z-score methodology. Briefly, for each questionnaire item, z-scores for individual respondents were calculated using the formula below, which indicates how many $\mathrm{SD}(\sigma)$ a rating $(\mathrm{X})$ is above or below the mean $(\mu)$ of the pooled data (all the respondents together).

$$
\mathrm{z}=(\mathrm{X}-\mu) / \sigma
$$

The directionality of the z-scores was adjusted so that higher score values reflected higher values for the subscale to which the questionnaire item belonged. The $\mathrm{z}$-scores for questionnaire items were first averaged within a

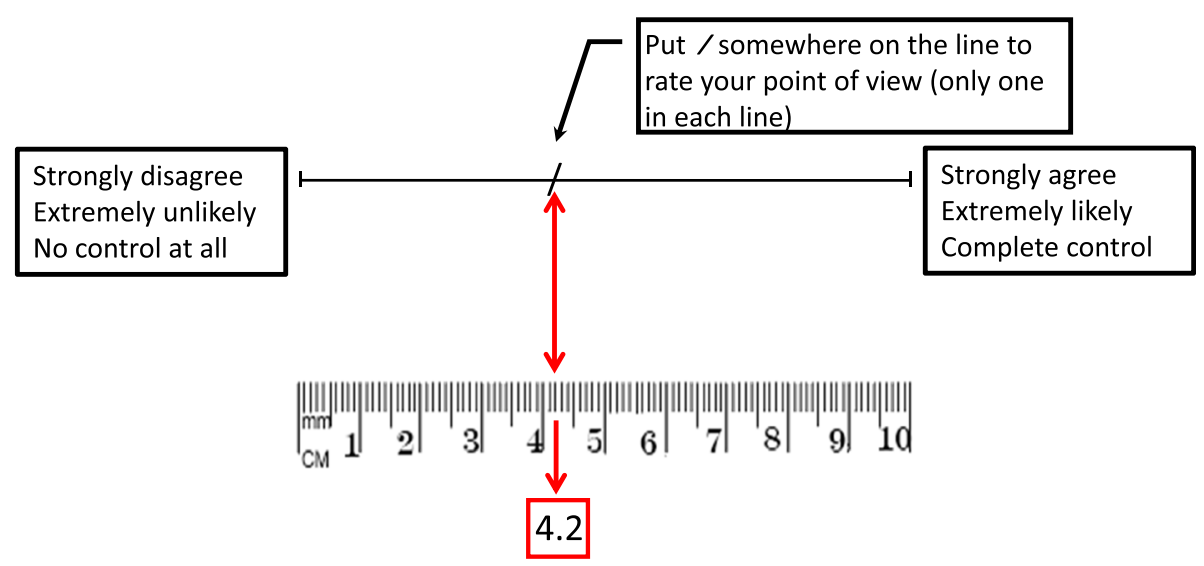

Fig. 1 Continuous rating scale used for parts 3 and 4 of the questionnaire (see Additional file 1: Appendix) 
subscale and then across subscales to ensure equal weighting of the subscales comprising the final combined $\mathrm{z}$-score for a specific scale.

\section{Statistical analyses}

All statistical analyses were carried out using an $\mathrm{IBM}^{\circ}$ SPSS $^{\circ}$ Statistics for Windows (version 22.0) computer program (IBM Corp., Armonk, NY, USA) and paying attention to the assumptions that underlie the various statistical procedures. Two-sided, exact (i.e., for the non-parametric tests) probabilities were estimated throughout. The categorical data from parts 1 and 2 of the questionnaire (see Additional file 1: Appendix) are presented as scores (number of cases) with in parentheses the relative frequency (\%). Many factors differ between the Dutch and Thai parts of the study, and in the statistical analyses some of these factors may form part of the factor culture/country whereas others, such as climate, may be confounding environmental factors. Log-linear models were used to analyse categorical data, to detect possible associations between the examined categorical variables and the categorical factors country (= Thailand or the Netherlands = culture $)$ and $B C S(=3,4$, or 5$)$.

The continuous data from parts 1 and 2 , as well as the integrated z-score data, are expressed as means with standard error of the mean (SEM). The Kolmogorov-Smirnov one-sample test was used (for continuous data) per group and revealed that some continuous variables were not normally distributed. The data for these variables were transformed to a Gaussian distribution, using a suitable mathematical function (logarithmic, logistic, square root or inverse transformation). If this was not possible, the data were rank transformed. Rank transformations are a bridge between parametric and non-parametric statistics [35].

Continuous data were compared with a two-way analysis of variance (ANOVA) with main factors country and BCS. An assumption for an ANOVA is that the residuals have a normal distribution. As some of the residuals were not normally distributed, the data were mathematically transformed (logarithmic, logistic, square root or inverse transformation). If this was not possible, the continuous data were rank transformed. For all ANOVAs, homoscedasticity was tested with Levene's test. When necessary, the variances were equalized by logarithmic, logistic, square root or inverse transformation. After transformation, the variances should be similar and the transformed within-group data should be normally distributed. If this was not the case, the data for the continuous variable in question were rank transformed [35]. An ANOVA performed on ranked data is also known as the Scheirer-Ray-Hare extension of the Kruskal-Wallis test [36].

As the dog's gender and sexual status, age, and duration of ownership may influence scores in parts 3 and 4 (see Additional file 1: Appendix), the integrated z-score data were also tested for significant differences by a four-way analysis of covariance (ANCOVA) with main factors country, BCS, dog's gender, and sexual status. Age of the dog and duration of dog ownership served as covariates. For the ANCOVAs, homoscedasticity was also tested with Levene's test.

Post hoc comparisons for normally distributed continuous data were performed with the unpaired Student's $t$ test. The Student's $t$ tests were performed using pooled (for equal variances) or separate (for unequal variances) variance estimates. The equality of variances was tested with Levene's test. For the unpaired Student's $t$ test with separate variance estimates, IBM $^{\bullet}$ SPSS $^{\circ}$ Statistics uses the Welch-Satterthwaite correction. Post hoc comparisons for non-normally distributed continuous data were performed with the Wilcoxon-Mann-Whitney test.

Spearman coefficient of rank correlation $\left(R_{S}\right)$ was calculated for: $i$ ) dog age and ownership; ii) dog age and BCS; iii) the $\mathrm{z}$-score for immobility and reported total activity (indoor plus outdoor); iv) dog age and z-score for general sickness. In addition Pearson's linear correlation coefficient $(r)$ was calculated for logarithmically transformed dog age $\left(y={ }^{10} \log [x+12]\right)$ and logarithmically transformed $z$-score for general sickness $\left(y={ }^{10} \log [x+0.7]\right.$. Significance was assessed by a two-tailed test based on the $t$ statistic.

In addition, the data from parts $3(\mathrm{QoL})$ and 4 (owner attitude) were also analysed by factor analysis, using a principal components solution with orthogonal rotation (varimax) of the factor matrix. This method ensures that the extracted factors are independent of one another and should, therefore, reflect separate processes. The varimax algorithm was chosen, because it attempts to minimize the number of variables that have high loadings (see hereafter) on a factor, which should improve interpretability. Interpretation of a factor analysis is only meaningful if all of the variables are assumed to be scaled at the numeric level (continuous scale of measurement or count based measures), which was the case for the answers to the questions of parts 3 and 4 of the questionnaire (see Processing of the questionnaires and z-score calculation). Our objective was data reduction and therefore principal component analysis was used instead of principal axis analysis [37].

Sampling adequacy was measured with the Kaiser-Meyer-Olkin measure (should be greater than $\approx 0.5$ ). The Bartlett's test of sphericity was used to test whether the correlation was appropriate for factor analysis. Factor pattern matrices were identified using a fixed number of factors (based on the number of subscales: 9 for QoL data and 11 for owner attitude data; or based on the number of subscales per scale: 2 for the psychological, social and environmental scales, 3 for the physical scale, 5 for the attitude in feeding scale and 6 for the attitude in exercise scale). All extracted factors had an eigenvalue $\geq 1$. The factor loading of each 
question indicated how well that question correlated with the factor; thus a loading of \pm 1.0 indicates a perfect (positive/negative) correlation, whereas an absolute loading of less than 0.4 would suggest that the question is rather weakly linked to the factor.

The highest (absolute) factor loadings based on total number of subscales (Tables 3 and 4) were compared with those based on number of subscales per scale (Additional file 1: Tables S3 and S4) using a paired Student's $t$ test, since the difference between the two compared sets of (absolute) factor loadings was normally distributed. Schneider et al. [12] conducted a principal axis factoring extraction with varimax rotation. (Absolute) factor loadings for QoL in the present study using a principal axis solution with varimax rotation (results not shown) were also compared (paired Student's $t$ test) to those reported by Schneider et al. [12]; again the difference between the two compared sets of (absolute) factor loadings was normally distributed.

To account for the greater probability of a Type I error due to multiple comparisons, a more stringent criterion should be used for statistical significance (i.e. for the Student's $t$ tests and Wilcoxon-Mann-Whitney tests). We approached this problem by calculating a so-called Dunn-Šidák correction $\left(\alpha=1-[1-0.05]^{1 / \gamma} ; \gamma=\right.$ number of meaningful comparisons; Tables 1 and 2, Additional file 1: Tables S1, S2, S5, S6 and S7 plus Figs. 2 and 3: $\gamma=9 \rightarrow \alpha=$ 0.005683 ). In all other cases, the probability of a Type I error $<0.05$ was taken as the criterion of significance.

\section{Results}

\section{General}

In total, 200 Thai dog owners and 155 Dutch dog owners completed the questionnaire. There were few missing answers (this information can be found in Tables 1 to 4, Additional file 1: Tables S1 to S6 plus Figs. 2 and 3), indicating that the owners experienced no difficulty in understanding/completing the questionnaire. Information about owner demographics, dog-feeding behaviour, and dog information can be found in Table 1 and Additional file 1: Table S1. Only sixteen respondents $(\approx 4.5 \%)$ were not totally or mostly ('child of owner' or 'caretaker') in charge of dog management (Additional file 1: Table S1).

Unfortunately, in the Netherlands it was very difficult to find dogs with a BCS of 5 that did not have another diseases besides obesity or overweight. There was no difference between BCS groups in the way the food was administered (log linear analysis interaction between 'quantity of food' and 'BCS': partial Chi-square $=10.621, d f=6, P=0.100809$ ), although Thai dogs were more often fed ad libitum than were Dutch dogs (log linear analysis interaction between 'quantity of food' and 'country': partial Chi-square $=47.842$, $d f=3, P<0.0000005^{*}$ ) (Table 1). Male, sexually intact Thai dogs had a BCS of 3 more often than did neutered Thai male dogs, and sterilized female Thai dogs more often had a BCS of 5 than did non-sterilized female Thai dogs (post hoc analysis, Fisher's Exact test: $\left.P=0.002622^{*}\right)$, but these differences were not seen in the Dutch dogs (post hoc analysis, Fisher's Exact test: $P=0.240207)$ (Table 1).

Table 1 Dog feeding behaviour and sexual status: categorical data ${ }^{1}$

\begin{tabular}{|c|c|c|c|c|c|c|c|}
\hline \multirow{3}{*}{$\begin{array}{l}\text { Country: } \\
\text { Measure (number of answers)/Category }\end{array}$} & \multicolumn{6}{|c|}{ Body condition score (BCS) } & \multirow[b]{3}{*}{ Log linear analysis significance } \\
\hline & \multicolumn{3}{|c|}{ The Netherlands } & \multicolumn{3}{|l|}{ Thailand } & \\
\hline & BCS 3 & BCS 4 & BCS 5 & BCS 3 & BCS 4 & BCS 5 & \\
\hline Quantity of food $(n=355)^{5}$ & $A^{4}$ & $\mathrm{~B}^{4}$ & & A & $B$ & & \\
\hline As it wants & $4(6.0 \%)^{2}$ & $5(8.1 \%)$ & $2(7.7 \%)$ & $29(43.9 \%)$ & $14(20.9 \%)$ & $26(38.8 \%)$ & \\
\hline As stated/calculated by veterinarian & $33(49.3 \%)$ & $29(46.8 \%)$ & $9(34.6 \%)$ & $13(19.7 \%)$ & $14(20.9 \%)$ & $14(20.9 \%)$ & \\
\hline As estimated & $30(44.8 \%)$ & $27(43.5 \%)$ & $15(57.7 \%)$ & $24(36.4 \%)$ & $38(56.7 \%)$ & $27(40.3 \%)$ & \\
\hline Do not know & $0(0.0 \%)$ & $1(1.6 \%)$ & $0(0.0 \%)$ & $0(0.0 \%)$ & $1(1.5 \%)$ & $0(0.0 \%)$ & $C, B, M, C \times B, M \times C$ \\
\hline Dog sexual status $(n=355)$ & A & & & $\mathrm{Aa}$ & & a & \\
\hline Male neutered & $14(20.9 \%)$ & $15(24.2 \%)$ & $11(42.3 \%)$ & $11(16.7 \%)$ & $13(19.4 \%)$ & $15(22.4 \%)$ & \\
\hline Male sexually intact & $20(29.9 \%)$ & $14(22.6 \%)$ & $6(23.1 \%)$ & $33(50.0 \%)$ & $22(32.8 \%)$ & $19(28.4 \%)$ & \\
\hline Female neutered & $20(29.9 \%)$ & $21(33.9 \%)$ & $5(19.2 \%)$ & $5(7.6 \%)$ & $15(22.4 \%)$ & $20(29.9 \%)$ & \\
\hline Female sexually intact & $13(19.4 \%)$ & $12(19.4 \%)$ & $4(15.4 \%)$ & $17(25.8 \%)$ & $17(25.4 \%)$ & 13(19.4\%) & $\mathrm{C}, \mathrm{B}, \mathrm{M}, \mathrm{C} \times \mathrm{B}, \mathrm{M} \times \mathrm{C}$ \\
\hline
\end{tabular}

${ }^{1}$ This table is based on part 1 and part 2 of the questionnaire (see Additional file 1: Appendix)

${ }^{2}$ Results are presented as scores (number of cases) with in parentheses the relative frequency (\%)

${ }^{3}$ Significance $(P<0.05)$ based on log linear analysis with categorical variables/factors measure (first column in this table), country and body condition score. $C$ indicates significant contribution of the factor country to the log linear model; B, significant contribution of the factor body condition score; $\mathrm{CxB}$, significant contribution of the interaction between the factors country and body condition score; MxB, significant contribution of the interaction between the variable measure and factor body condition score; $\mathrm{MxC}$, significant contribution of the interaction between the variable measure and factor country

${ }^{4}$ Contrast significance (post hoc comparisons, $P<0.005683$ ). Post hoc testing was done by Fischer's Exact test. Within the same country values with the same superscript lowercase letter were significantly different. Within the same category of body condition score values with the same superscript uppercase letter were significantly different

${ }^{5}$ The number of answers is given in parentheses 
Table 2 Owner demographics, dog information and activities: continuous data ${ }^{1}$

\begin{tabular}{|c|c|c|c|c|c|}
\hline \multirow{2}{*}{$\begin{array}{l}\text { Measure } \\
\text { (number of answers) }^{5}\end{array}$} & \multirow[b]{2}{*}{ Country } & \multicolumn{3}{|c|}{ Body condition score (BCS) } & \multirow[b]{2}{*}{ Transformation/ANOVA significance 3} \\
\hline & & $\mathrm{BCS} 3$ & BCS 4 & BCS 5 & \\
\hline \multicolumn{6}{|l|}{ Owner age (years) } \\
\hline \multirow[t]{2}{*}{$(n=355)$} & Netherlands & $42.0 \pm 1.9^{2}$ & $44.1 \pm 1.8$ & $47.8 \pm 3.4$ & Ranking \\
\hline & Thailand & $37.6 \pm 1.4$ & $38.5 \pm 1.6$ & $38.2 \pm 1.5$ & C \\
\hline \multicolumn{6}{|l|}{ Ownership (years) } \\
\hline \multirow[t]{2}{*}{$\left(n=354^{*}\right)$} & Netherlands & $5.4 \pm 0.5$ & $5.5 \pm 0.5$ & $7.3 \pm 0.6$ & Ranking \\
\hline & Thailand & $3.9 \pm 0.2^{\mathrm{a} 4}$ & $4.7 \pm 0.2$ & $5.3 \pm 0.2^{a}$ & $C, B$ \\
\hline \multicolumn{6}{|l|}{ Dog age (years) } \\
\hline \multirow[t]{2}{*}{$(n=355)$} & Netherlands & $5.8 \pm 0.5^{\mathrm{A}}$ & $6.2 \pm 0.5$ & $8.2 \pm 0.7^{B}$ & Ranking \\
\hline & Thailand & $4.2 \pm 0.2^{\mathrm{aA}}$ & $5.1 \pm 0.2^{b}$ & $5.6 \pm 0.2^{\mathrm{abB}}$ & $C, B, C \times B$ \\
\hline \multicolumn{6}{|c|}{ Total indoor activity (h/week) } \\
\hline \multirow[t]{2}{*}{$\left(n=353^{*}\right)$} & Netherlands & $8.9 \pm 1.4^{\mathrm{aA}}$ & $5.3 \pm 1.0^{B}$ & $3.7 \pm 1.7^{\mathrm{ac}}$ & Ranking \\
\hline & Thailand & $47.9 \pm 4.4^{\mathrm{A}}$ & $41.3 \pm 4.4^{B}$ & $40.1 \pm 5.5^{c}$ & $C, B, C \times B$ \\
\hline \multicolumn{6}{|c|}{ Total outdoor activity (h/week) } \\
\hline \multirow[t]{2}{*}{$\left(n=353^{*}\right)$} & Netherlands & $19.7 \pm 1.7$ & $18.3 \pm 1.8^{\mathrm{A}}$ & $20.8 \pm 3.7$ & Ranking \\
\hline & Thailand & $21.1 \pm 3.3$ & $13.1 \pm 2.1^{\mathrm{A}}$ & $20.5 \pm 3.8$ & $C$ \\
\hline \multicolumn{6}{|c|}{ Total indoor plus outdoor activity (h/week) } \\
\hline \multirow[t]{2}{*}{$\left(n=352^{*}\right)$} & Netherlands & $28.6 \pm 2.7^{\mathrm{A}}$ & $23.6 \pm 2.3^{B}$ & $24.5 \pm 4.1^{c}$ & Ranking \\
\hline & Thailand & $69.0 \pm 5.7^{\mathrm{A}}$ & $54.4 \pm 4.4^{B}$ & $61.6 \pm 8.2^{c}$ & $C, B$ \\
\hline
\end{tabular}

${ }^{1}$ This table is based on part 1 and part 2 of the questionnaire (see Additional file 1: Appendix)

${ }^{2}$ Results are presented as means \pm SEM

${ }^{3}$ Significance $(P<0.05)$ based on two-way ANOVA with main factors country and body condition score. $C$ indicates effect of country; B, effect of body condition score; $\mathrm{CxB}$, interaction. Measures that are not normally distributed and/or where the variances were unequal were first transformed. In this column the type of transformation is also given

${ }^{4}$ Contrast significance (post hoc comparisons, $P<0.005683$ ). Post hoc testing was done by unpaired Student's $t$ test (Gaussian distributed data + homoscedasticity), unpaired Student's $t$ test with Welch-Satterthwaite correction (Gaussian distributed data + heteroscedasticity) or Wilcoxon-Mann-Whitney test (non-Gaussian distributed data). Within the same row (country) values with the same superscript lowercase letter were significantly different. Within the same column (body condition score) values with the same superscript uppercase letter were significantly different

${ }^{5}$ The number of answers is given in parentheses

* Indicates missing answers

Among the Thai dogs, there was a significant difference in years of ownership between the dogs in BCS groups 3 and 5 (post hoc analysis, unpaired Student's $t$ test, $t_{131}=$ $\left.-4.032, P=0.000093^{*}\right)$. As years of ownership was significantly $\left(P<0.0000005^{*}\right)$ correlated with dog age in Thailand (Additional file 1: Figure S1, panel A: $R_{S}=0.898$ ), the older the dog the higher its BCS score tended to be (see Table 2 and Additional file 1: Figure S2: $\left.R_{S}=0.302, P=0.000014^{*}\right)$. A similar trend was seen among Dutch dogs (Additional file 1: Figure S1, panel B: $R_{S}=0.906, P<0.0000005^{*}$; Additional file 1: Figure S2: $R_{S}=0.193, P=0.016343^{*}$ ). When looking at the effect of BCS on exercise, we distinguished between indoor (i.e. in house) and outdoor exercise. Although we thought that dogs in Thailand in general would have less outdoor exercise than dogs in the Netherlands, this was only the case for dogs in BCS group 4, based on the total outdoor activity score (Table 2; post hoc analysis, Wilcoxon-Mann-Whitney test: $U=1333.5, W$ $\left.=3611.5, Z=-3.519, P=0.000375^{*}\right)$. However, the total indoor activity of Thai dogs was significantly higher than that of Dutch dogs (Table 2; ANOVA country: rank-transformed, $\left.F_{1,347}=11.935, P=0.000619^{*}\right)$, so that the Thai dogs had more exercise overall (Table 2; ANOVA country: rank-transformed, $\left.F_{1,346}=92.736, P<0.0000005^{*}\right)$. In post hoc comparisons no significant differences (unpaired Student's $t$ test or Wilcoxon-Mann-Whitney test: $P$ $\geq 0.005683$ ) were found in overall exercise between the three BCS groups (Table 2). In the Dutch cohort, dogs in the BCS 5 group had significantly less indoor exercise than dogs in the BCS 3 group (post hoc analysis, Wilcoxon-Mann-Whitney test: $U=481.0, W=832.0, Z=$ $\left.-3.388, P=0.000559^{*}\right)$, but this difference was not seen in the Thai cohort (post hoc analysis, Wilcoxon-Mann-Whitney test: $U=1666.5, W=3810.0, Z=-2.209, P=0.026942$ ) (Table 2). There were some differences between BCS groups in the type of exercise; these results are presented in Additional file 1: Table S2.

\section{Orthogonal factor loadings}

Principal component factor analysis was used to analyse the owner-reported QoL data (Table 3). Nine factors from the 34 questions accounted for $55.0 \%$ of the total variance, 
a

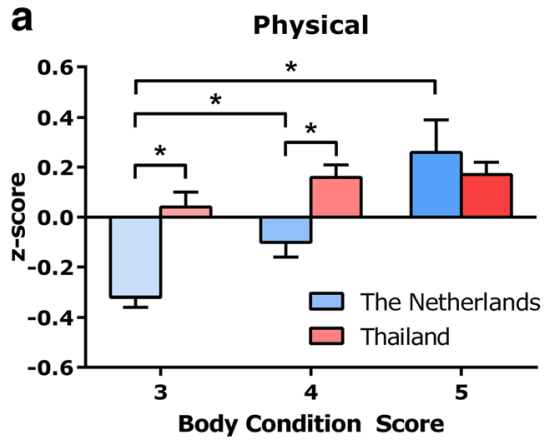

ANOVA $\left(n=353^{\star}\right) / \operatorname{ANCOVA}\left(n=352^{\star}\right):$ C, B, CXB; ranking

C

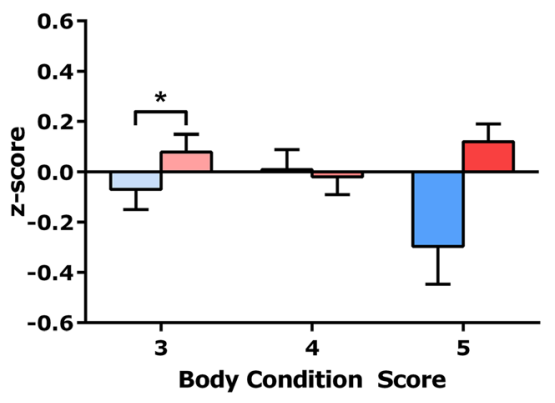

b

Psychological

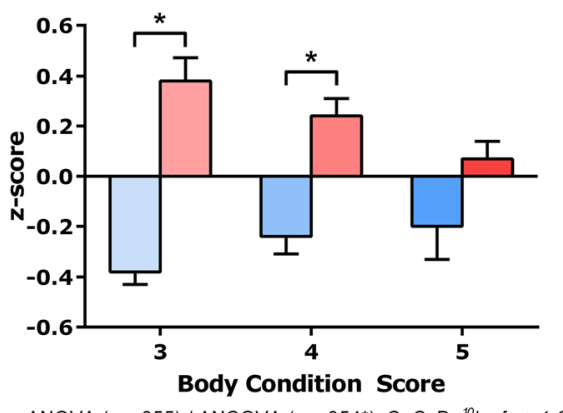

ANOVA $(n=355) /$ ANCOVA $\left(n=354^{*}\right)$ : C, CxB; ${ }^{10} \log [x+1.6]$

d

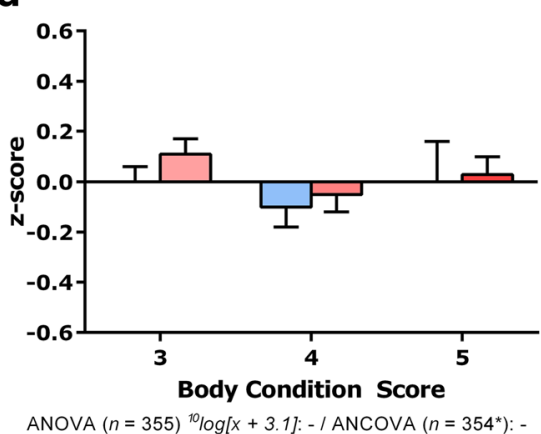

Fig. 2. Owner reports of quality of life: scale z-scores. This figure is based on part 3 of the questionnaire (see Additional file 1: Appendix) and the subscale z-scores summarized in Additional file 1: Table S5. Panel a, Physical; panel b, Psychological; panel c, Social; panel d, Environmental. The subscale z-scores were averaged, resulting in a scale $z$-score. Results are presented as means \pm SEM. ANOVA = two-way analysis of variance with main factors country and body condition score. $C$ indicates effect of country; B, effect of body condition score; $C \times B$, interaction; $-=$ no significant $C$, B and CxB effect. Effects were significant when $P<0.05$. Scale $z$-scores that were not normally distributed and/or where the variances were unequal, were first transformed. In the figure the type of transformation is indicated. The data were also tested for significant $(P<0.05)$ differences by an ANCOVA with main factors country and body condition score, dog's gender and sexual status. Covariates were age of the dog and duration of ownership. Post hoc testing was done by unpaired Student's $t$ test (Gaussian distributed data + homoscedasticity), unpaired Student's $t$ test with Welch-Satterthwaite correction (Gaussian distributed data + heteroscedasticity) or Wilcoxon-Mann-Whitney test (non-Gaussian distributed data). ${ }^{*}=$ Significant difference $(P<0.005683)$ in the post hoc comparison

a

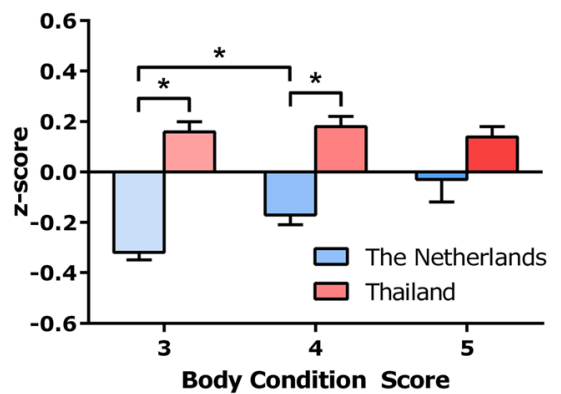

ANOVA $\left(n=353^{*}\right)$ : C, B, CxB / ANCOVA $\left(n=352^{*}\right)$ : C, CxB; ranking

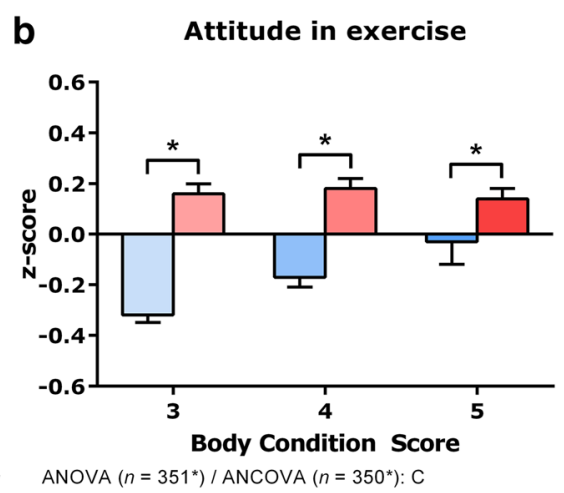

Fig. 3 Owner attitude: scale z-scores. This figure is based on part 4 of the questionnaire (see Additional file 1: Appendix) and the subscale z-scores summarized in Additional file 1: Table S6. Panel $\mathbf{a}$, Attitude in feeding; panel $\mathbf{b}$, Attitude in exercise. The subscale z-scores were averaged, resulting in a scale $z$-score. Results are presented as means \pm SEM. ANOVA = two-way analysis of variance with main factors country and body condition score. $\mathrm{C}$ indicates effect of country; B, effect of body condition score; CxB, interaction; - = no significant C, B and CxB effect. Effects were significant when $P<0.05$. Scale $z-$ scores that were not normally distributed and/or where the variances were unequal, were first transformed. In the figure the type of transformation is indicated. The data were also tested for significant differences by an ANCOVA with main factors country and body condition score, dog's gender and sexual status. Post hoc testing was done by unpaired Student's $t$ test (Gaussian distributed data + homoscedasticity), unpaired Student's $t$ test with WelchSatterthwaite correction (Gaussian distributed data + heteroscedasticity) or Wilcoxon-Mann-Whitney test (non-Gaussian distributed data). ${ }^{*}=$ Significant difference $(P<0.005683)$ in the post hoc comparison 


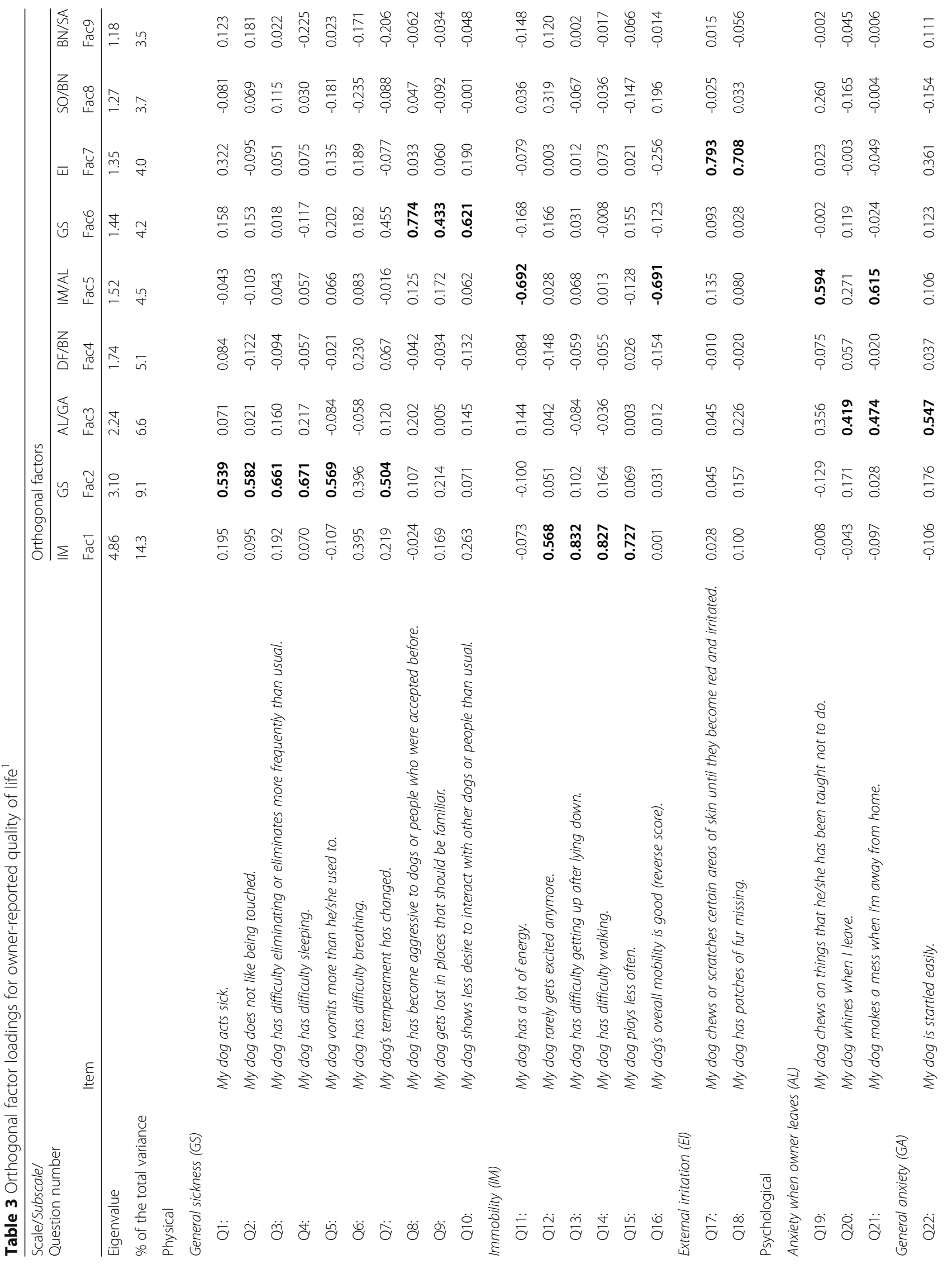




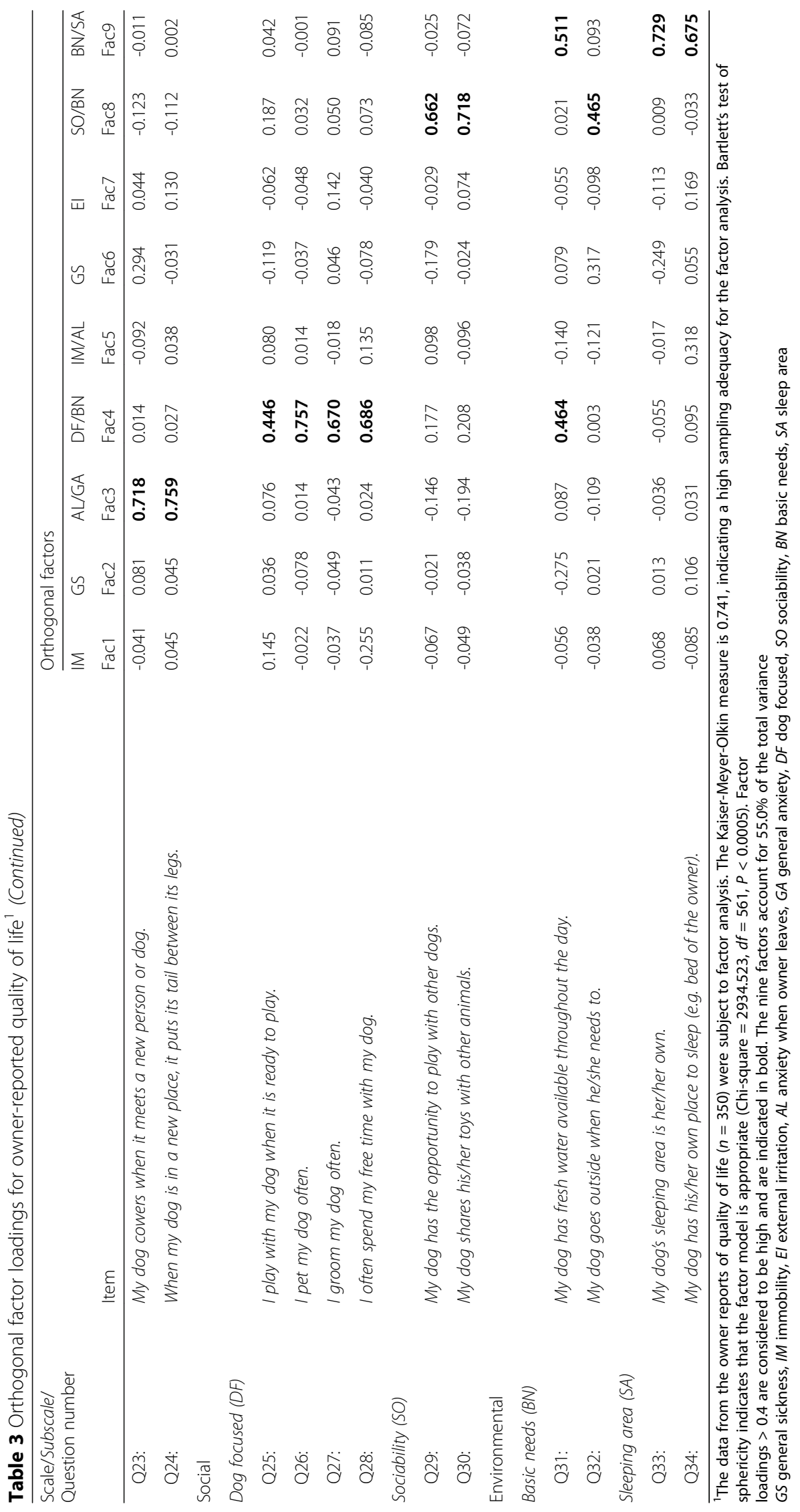


with eigenvalues $>1.17$. The psychological subscale 'Anxiety when the owner leaves' and the environmental subscale 'Basic needs' had somewhat low reliability and hence interpretations require some caution. Additional file 1: Table S3 summarizes the results of the principal component factor analyses for owner-reported QoL data, but now per scale, which - as would be expected - clearly improved the reliability of the subscales 'Anxiety when the owner leaves' and 'Basic needs'. However, there was no significant difference in the magnitude of the highest (absolute) factor loading (per question) between data presented in Table 3 and Additional file 1: Table S3 (paired Student's $t$ test, $t_{33}=-1.221, P=0.230780$ ).

The orthogonal factor loadings for owner attitude are shown in Table 4. The 11 factors extracted from the 42 questions accounted for $72.0 \%$ of the total variance. The attitude in exercise-subscale 'Owner centred' had somewhat low reliability and hence interpretation requires some caution. Additional file 1: Table S4 shows the results of the principal component factor analyses per scale for owner attitude, which improved the reliability of the subscale 'Owner centred'. On average, the (absolute) factor loadings of data in Table 4 were 1.8 times (and significantly) higher than those of data in Additional file 1: Table S4 (paired Student's $t$ test, $\left.t_{41}=-2.269, P=0.028580^{*}\right)$.

\section{Quality of life}

Because we included only healthy dogs, we did not expect to find differences in QoL scale 'Physical' between the BCS groups (Additional file 1: Table S5). However, among the Dutch dogs, BCS was positively associated with 'General sickness', indicating that according to Dutch owners overweight and obesity influenced the health status of their dogs (ANOVA, logarithmically transformed: country, $F_{1,348}=$ $8.508, P=0.003765^{*} ;$ body condition score, $F_{2,348}=10.266$, $P=0.000047^{*}$; interaction, $\left.F_{2,348}=3.136, P=0.044669^{*}\right)$. The Dutch dogs with a BCS of 3 had lower scores for 'General sickness' than their Thai counterparts (post hoc analysis, Wilcoxon-Mann-Whitney test: $U=1346.5, W=$ 3624.5, $\left.Z=-3.892, P=0.000079^{*}\right)$. Dog immobility increased with increasing BCS (ANOVA body condition score: rank-transformed, $\left.F_{1,348}=21.098, P<0.0000005^{*}\right)$. This effect was more pronounced in the Dutch dogs (ANOVA interaction: rank-transformed, $F_{2,348}=11.432, P=$ $0.000016^{*}$ ): Thai dogs with a BCS of 5 were less immobile than Dutch dogs with the same BCS (post hoc analysis, unpaired Student's $t$ test with Welch-Satterthwaite correction, $\left.t_{28.484}=-3.115, P=0.004168^{*}\right)$, whereas the opposite was true for dogs with a BCS of 3 (post hoc analysis, Wilcoxon-Mann-Whitney test: $U=1148.5, W=3426.5, Z$ $\left.=-4.782, P<0.0000005^{*}\right)$.

Two aspects of anxiety were included in the scale 'Psychological', 'Anxiety when owner leaves' and 'General anxiety' (Additional file 1: Table S5). The Thai dogs were more anxious when their owner left home than were the Dutch dogs (ANOVA country: rank-transformed, $F_{1,349}=12.859$, $\left.P=0.000384^{*}\right)$, but this difference was only significant for dogs with a BCS of 3 (post hoc analysis, Wilcoxon-Mann-Whitney test: $U=955.5, W=3233.5, Z=$ $-5.655, P<0.0000005^{*}$ ) or a BCS of 4 (post hoc analysis, Wilcoxon-Mann-Whitney test: $U=1201.5, W=3154.5, Z$ $\left.=-4.131, P=0.000026^{*}\right)$. In the Netherlands, the group mean for 'Anxiety when owner leaves' increased with increasing BCS, whereas it decreased in Thailand, suggesting that there was an interaction effect of country and BCS (ANOVA interaction: rank-transformed, $F_{2,349}=8.288, P=$ $\left.0.000304^{*}\right)$. On the social and environmental scales (Additional file 1: Table S5), only the factor 'country' influenced QoL ('Dog focused', ANOVA country: non-transformed, $F_{1,348}=11.644, P=0.000720 *$; 'Sociability', ANOVA country: non-transformed, $F_{1,347}=0.636, P=$ 0.425725; 'Basic needs', ANOVA country: rank-transformed, $F_{1,349}=8.378, P=0.004036^{*}$; 'Sleeping area', ANOVA country: non-transformed, $F_{1,349}=12.975, P=0.000361^{*}$ ).

The data were also tested for significant differences using an ANCOVA with main factors country, BCS, dog's gender, and sexual status; covariates were dog age and duration of ownership. Country, and an interaction between country and $B C S$, influenced 'General anxiety' (ANCOVA, rank-transformed: country, $F_{1,328}=43.0565, P<0.0000005^{*}$; interaction, $F_{2,328}=3.183, P=0.042737^{*}$ ) and 'Sleeping area' (ANCOVA, rank-transformed: country, $F_{1,328}=6.050, P=$ $0.014423^{*}$; interaction, $\left.F_{2,328}=9.437, P=0.000104^{*}\right)$. There was now a main effect of BCS on 'Sleeping area' (ANCOVA, rank-transformed: body condition score, $F_{2,328}=4.395, P=$ $0.013068^{*}$ ) (Additional file 1: Table S5).

Combination of the QoL z-scores identified four scales, namely, Physical, Psychological, Social, and Environmental (Fig. 2). In the Dutch cohort, physical scale scores differed by BCS group, with the higher the BCS, the higher the physical scale score (post hoc analyses, unpaired Student's $t$ test: BCS 3 versus BCS 4, Welch-Satterthwaite correction, $t_{108.616}=-3.721, P=$ $0.000316^{*} ;$ BCS 3 versus BCS 5, Welch-Satterthwaite correction, $t_{28.457}=-4.837, P=0.000042^{*} ;$ BCS 4 versus BCS 5, no correction, $\left.t_{84}=-2.812, P=0.006124\right)$.

\section{Owner attitude}

Country, BCS, and an interaction on all the subscales significantly (ANOVA, rank-transformed, $P<0.05^{*}$ ) influenced 'attitude in feeding' (Additional file 1: Table S6). The Dutch respondents rated their control of feeding higher than did the Thai respondents, with the owners of dogs with a BCS of 3 reporting the highest control over feeding (ANOVA rank-transformed: country, $F_{1,349}=12.985, P=0.000360^{*}$; body condition score, $F_{2,349}=7.830, P=0.000471^{*}$; interaction, $F_{2,349}=8.369, P=0.000282 \%$ ). The Thai owners fed their dogs to please more often than did the Dutch 


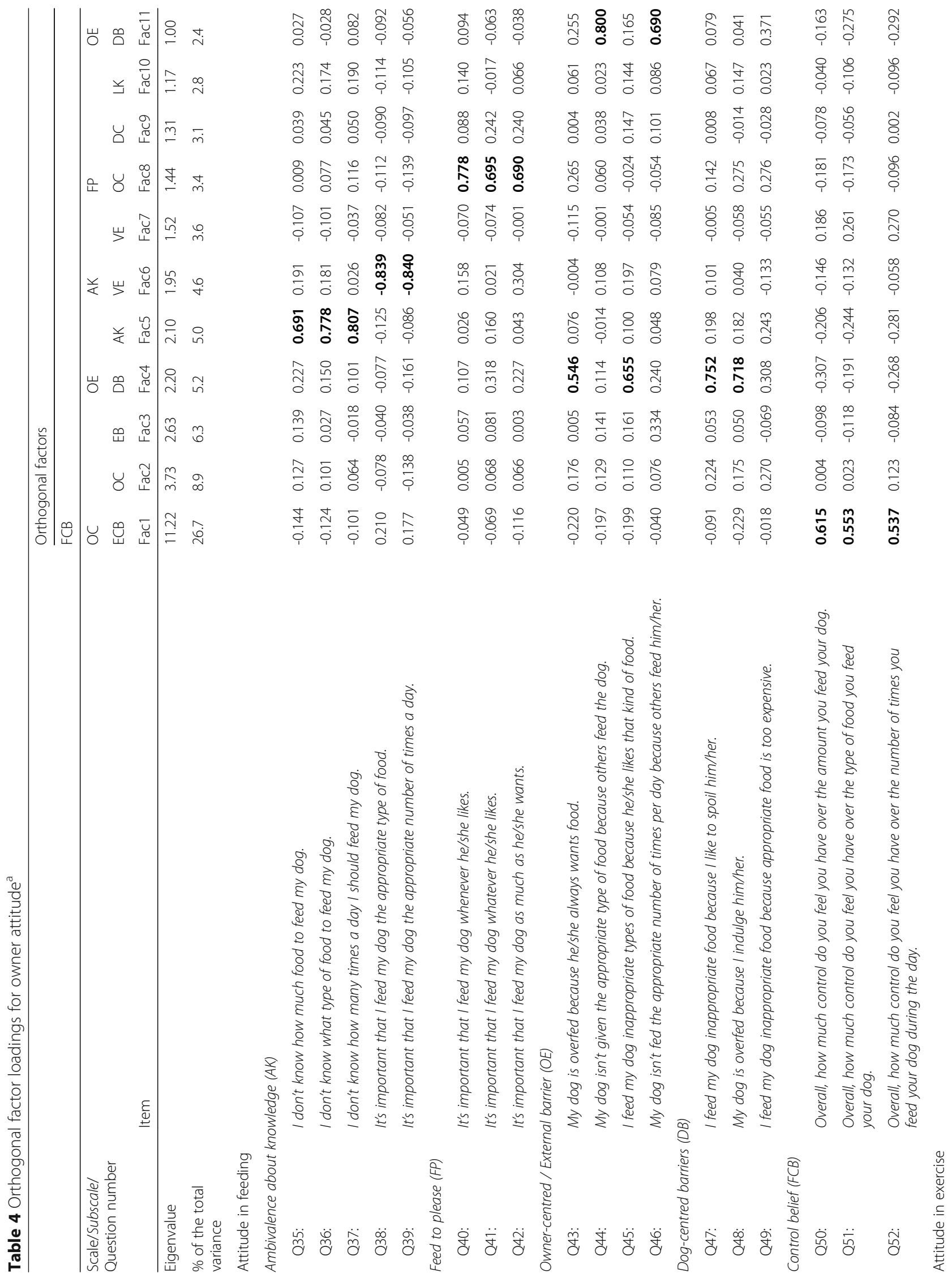




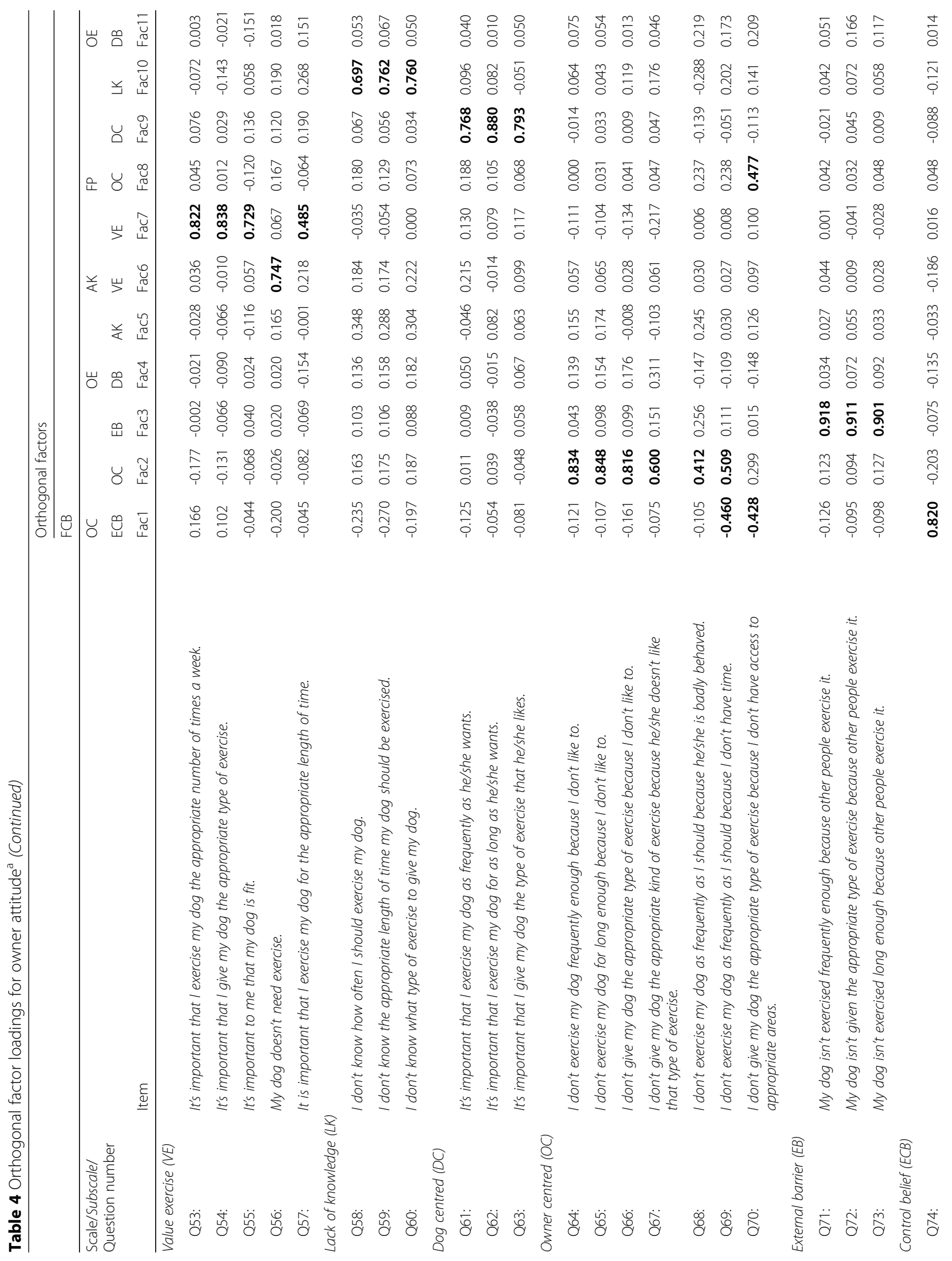




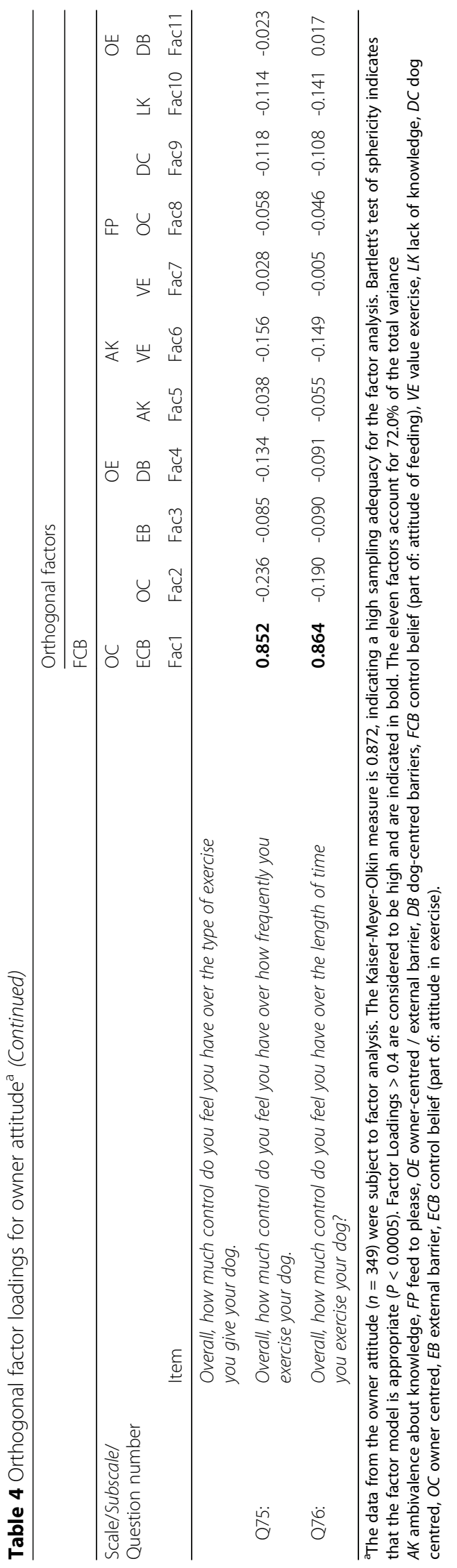


owners (ANOVA country: rank-transformed, $F_{1,349}=12.865$, $P=0.000383 *)$. This difference was significant for BCS 3 (post hoc analysis, Wilcoxon-Mann-Whitney test: $U=690.0$, $W=2968.0, Z=-6.857, P<0.0000005^{*}$ ) and BCS 4 (post hoc analysis, unpaired Student's $t$ test, no correction, $t_{127}=$ $-4.028, P=0.000096$ ) dogs.

Only in the Netherlands did the subscales 'value of exercise' (post hoc analysis, BCS 3 versus BCS 5, Wilcoxon-Mann-Whitney test: $U=473.0, W=824.0, Z$ $\left.=-3.418, P=0.000494^{*}\right)$, 'lack of knowledge about exercise' (post hoc analysis, BCS 3 versus BCS 5, Wilcoxon-Mann-Whitney test: $U=504.5, W=2782.5$, $\left.Z=-3.156, P=0.001356^{*}\right)$, and the fact that owners were more or less self-centred' (post hoc analysis, BCS 3 versus BCS 5 , Wilcoxon-Mann-Whitney test: $U=411.5$, $\left.W=2689.5, Z=-3.940, P=0.000049^{*}\right)$ significantly affect BCS (Additional file 1: Table S6). As would be expected, in the Netherlands groups mean scores for 'value of exercise' decreased with increasing BCS of the dogs.

Z-scores for the 'attitude' subscales were combined to yield two scales, i.e. 'Attitude in feeding' and 'Attitude in exercise' (Fig. 3). 'Attitude in feeding' was significantly different between the Dutch owners of dogs with a BCS of 3 and 4 (post hoc analysis, Wilcoxon-Mann-Whitney test: $U$ $=1261.5, W=3472.5, Z=-3.627, P=0.000240 *)$, and there was a significant between-countries difference in 'attitude in exercise' (ANOVA country: non-transformed, $\left.F_{1,345}=32.690, P<0.0000005^{*}\right)$. The association between BCS and 'attitude in feeding' disappeared on ANCOVA (ANOVA rank-transformed: body condition score, $F_{2,347}=$ 4.894, $P=0.008018^{*}$; ANCOVA rank-transformed: $b o d y$ condition score, $\left.F_{2,326}=1.611, P=0.201229\right)$.

\section{Discussion}

\section{General}

Both the Dutch and Thai questionnaires were designed to be completed by owners while they were waiting for the veterinarian or dog groomer, and most owners were willing to do so. In the Netherlands, it was very difficult to find dogs that were 'healthy' despite being overweight or obese (BCS 4/5) and we recruited only 26 dogs with a BCS of 5. Moreover, the Dutch dog owners were less homogeneous than the Thai owners, possibly because the Thai owners were recruited at one site (KU-VTH), whereas in the Netherlands owners were recruited at various veterinary practices or dog grooming parlours in the province of Utrecht.

There were a number of potential confounders, such as differences in climate. In the Netherlands there is a temperate maritime climate with cool summers and moderate winters. Since the country is small there is little variation in climate from region to region. There are 4.5 million inhabitants in the province of Utrecht, whereas there are more than 10 million inhabitants in the metropolis of Bangkok, where the climate is tropical, with high temperature and high humidity. These climatic factors certainly influence the way dogs are kept, how often they are exercised outdoors, etc. For instance, obese dogs may be less tolerant of heat, which may affect the QoL of dogs living in warmer climates [38]. Homes with housekeepers or maids are much more common in Thailand than in the Netherlands. As these people also take care of the dogs, it is possible that the owners do not have all the information about their dog. These confounding factors probably influenced the results and were not taken into account. The reader should bear this in mind.

Body condition was scored with the BCS card of Hill's Pet Nutrition. Witzel et al. [39] found that morphometric measurements and the body fat index to be more accurate than the 5-point BCS method, but recognized that their approach needed to be validated in other dogs, such as normal and underweight dogs. There are other scoring systems, such as the 7-point SHAPE algorithm. This scoring system has been validated and owners are able to use it without prior training [40]. In the Netherlands, veterinarians work with the 5-point BCS, and the veterinarian who used the BCS in Thailand had been trained to use it in the Netherlands. In this way, we hoped to obtain a good inter-observer reliability. The SHAPE algorithm could be used in future studies.

In Thailand, dog sexual status significantly affected the BCS: intact dogs typically had a BCS of 3 whereas spayed and neutered dogs typically had a BCS of 5 (Table 1). There is strong evidence that dogs gain weight after sterilization ([41] and references cited therein), possibly because they are less active (but with the same energy intake) or they eat more [42]. Moreover, BCS increased with dog age, which was correlated with the duration of dog ownership: the older the dog, the longer it was owned, and the higher the BCS (Table 1). This is not surprising since overweight and obesity is considered a disorder of the middle-aged dogs [41].

\section{Limitations of translating a questionnaire in a cross- cultural setting}

Questionnaires are widely used in veterinary research [43] and generally provide a low-cost method to obtain information about a range of factors. With careful design, assessment and administration, questionnaires can collect accurate data. In cross-cultural settings with respondents that speak different languages - like this study - questionnaire design is complicated by the added step of translation. One of the major problems with cross-cultural research is to ensure that the questions are comparable across cultural groups and that interpretations are not affected by cultural bias. For example wording of questions might have to be changed 
to ensure comparability of meaning, especially as some words and concepts in one language will have no equivalent in another language. Guidelines exist for designing questionnaires in a cross-cultural setting (see: http:// ccsg.isr.umich.edu/index.php/chapters/questionnaire-design-chapter). Regarding translation equivalence there is no gold standard, but variations of Brislin's classic and iterative back-translation model are most commonly used to check the accuracy of questionnaire translation in cross-cultural studies [44]. Ideally this would briefly imply for the present study that: $i$ ) a trilingual translator (a competent veterinarian with expertise in the field) translates the questionnaire from the original, source language (here: English) into the two target languages (here: Dutch and Thai); ii) another trilingual translator (again a competent veterinarian with expertise in the field) translates the two versions back into English; iii) the three English versions of the questionnaire (original and two back-translated versions) are compared for equivalence; iv) items with apparent discrepancies between the three English versions are then modified; v) the translation/back-translation process is repeated by individual trilingual translators until the original and back-translated versions agreed.

In our study this wasn't done because at that time trilingual translators, who are veterinarians with appropriate knowledge of the topic, could not be found due to time constrains (if they exist at all). The translation of the English questionnaire to the Thai and Dutch language was done by native speakers in Thai and Dutch who were fluent in English, but not by people who were strictly bilingual as advised by Chen and Boore [33]. They recommend that "the translator is fluent in both the source language and target language and is knowledgeable about both cultures". Although the translators knew about the human-animal bond and/or had knowledge about the foreign language, the use of non-bilingual translators could have influenced the validity of the questionnaire [34]. However the questionnaires were satisfactorily pre-tested in the target population, which tends to support their validity [34]. But at this stage it is advisable to use the translated questionnaires cautiously.

A possibility would have been to split the results into a Thai part and a Dutch part and describe and discuss the results separately. By taken the Thai and Dutch samples together there is a more powerful experimental design (two-factor design) than with a one-factor design per culture. For this reason and because culture comparisons are interesting, it was decided to do not split the results.

For future cross-cultural studies (with original English questionnaires) involving two non-English speaking countries (e.g. the Netherlands and an Asiatic country) an alternative translation technique might be advisable: the decentering approach [45]. This is a process in which (bilingual) translators move back and forth amongst the languages, checking for cultural and linguistic accuracy. In this way we may prevent that people from the East and the West interpret the questions in different ways because of semantics and cultural differences, when answering questions. It is possible that dog owners in Thailand gave more social desirable answers than owners in the Netherlands. However the translation into Thai and Dutch was done by people living in these cultures.

\section{Likert scales versus visual analogue scales}

The Likert scales used in the original questionnaires of Schneider et al. [12] (5-point scale) and Rohlf et al. [6] (7-point scale) provide ordinal data and so non-parametric tests should be used, which have less power than parametric tests. Furthermore, ordinal scales limit the choice of subsequent (non-parametric) statistical analyses. For this reason, together with the ability to calculate $\mathrm{z}$-scores and perform standard linear principal component analyses, we preferred to use continuous scales (visual analogue scales; see Additional file 1: Appendix, parts 3 and 4). Few studies have compared itemized rating scales with continuous rating scales [46-49], but on the basis of these studies we feel that using a continuous rating scale or a Likert type scale does not affect validity. For example, Lange \& Söderlund [46] reported that there were no systematic differences between the two scales. However, changing the scale of a questionnaire from categorical to continuous is not without consequences. While McKelvie [49] found that respondents preferred continuous rating scales, these scales were more tiring to use, which could influence the results. McKelvie commented that with smaller numbers of categories there is a loss of discriminative power and values for $r$ in correlations, but that with larger numbers of categories, as in our study, a continuous measure does not have psychometric disadvantages [49]. For the moment, there is not enough evidence to conclude that one scale type is psychometric better than the other [50]. Thus we feel, based on Mckelvie [49], that changing the original rating scale of Schneider et al. [12] and Rolhf et al. [6] to a continuous rating scale did not have psychometric consequences. Despite changing the score scale, after a principal axis factoring extraction with varimax rotation (like Schneider et al. [12]), we found a similar magnitude of (absolute) factor loadings for QoL as in Study 2 (ill dogs) of Schneider et al. [12] (Additional file 1: Table S7), suggesting that part 3 of the questionnaire (Additional file 1: Appendix) can also be used by the owners of overweight or obese dogs and is not dependent on the cultural background of the respondent. This probably reflects the fact that obesity is a prevalent illness [22], and that animals with overweight are more likely to become obese. The factor loadings in 
Study 1 (healthy dogs) were higher than those in Study 2 (ill dogs) in the publication of Schneider et al. [12], and higher than those in our study (paired Student's $t$ test, $P$ $\left.<0.005683^{*}\right)$. However, the inferential statistical results from Additional file 1: Table S7 should be interpreted with caution. In Schneider et al. [12], items were rated using a 5-point Likert-type scale whereas we used a continuous scale. The most common extraction methods used in factor analysis are principal component analysis and principal axis analysis, which usually result in similar solutions [51]. However, we found that the magnitude of the factor loadings for QoL after principal component analysis were higher than those after principal axis analysis (Additional file 1: Table S7; paired Student's $t$ test, $\left.P<0.005683^{*}\right)$.

\section{Human-dog bond}

We did not take the human-animal bond into account because of time restraints - most owners completed the questionnaire while waiting for the veterinarian or waiting for their dogs at a grooming parlour. Although aspects of the human-animal bond influence how owners rate the health of their dogs [12], incorporating these aspects would have made the questionnaire too long, which might have increased the (item) non-response rate, thereby decreasing reliability. The original questionnaire of Schneider et al. [12] was designed for a normal, healthy dog population and an unhealthy, ill dog population in which $7.3 \%$ of the dogs were obese.

\section{QoL}

On the basis of owner report, dogs with a BCS of 5 were more immobile than dogs with a BCS of 3 , and this effect was more significant in the Dutch cohort than in the Thai cohort (Additional file 1: Table S5). There was no clear association between the z-score for immobility (Additional file 1: Table S5) and the reported total activity (Table 2): for individual respondents $(n=351)$ the $R_{S}=-0.045$ and the associated $P=0.397146$ for these two measures. Although we thought that Thai dogs would have less exercise outdoors than the Dutch dogs, this was not the case. It is possible that, in the Netherlands, dogs run free and are not on the leash [52], which might make it difficult for owners to estimate how much exercise their dogs have outdoors. It is also possible that the owners of dogs with a BCS of 4 or 5 gave socially desirable answers, saying that their dogs had a lot of exercise [53].

The observation that owners scored the general sickness component of QoL significantly higher (i.e., worse) when dogs had a higher BCS is surprising, because the dogs that participated in the study were overweight but otherwise healthy. There was also a weak but significant correlation between age of the dog and general sickness ('dog age' vs. 'z-score general sickness': $R_{S}=0.169, P=0.001442, n=354$; 'transformed dog age' vs. 'transformed z-score general sickness': $r=0.200, P=0.000155, n=354)$. Pearson's linear correlation coefficient showed that only $4 \%$ of the variation in general sickness could be explained by the age of the dog. German et al. [2] reported that QoL improved as dogs lost weight. Psychological, social, and environmental components were not influenced by BCS (Fig. 2), but it is possible that owners found it difficult to assess these QoL aspects.

The questionnaire item (see Additional file 1: Appendix) 'Anxiety when owner leaves' maybe synonymous with 'separation anxiety'. In fact, the term 'separation-related problems' is preferable to 'separation anxiety' when trying to describe the problematic behaviour seen when a dog is left alone, because the latter implies that a diagnosis has been made [54]. For this reason, we should have used the term 'separation-related problems'. Even so, the Thai dogs showed more anxiety/separation-related problems than did Dutch dogs when left alone at home. This is difficult to explain but it could be that Thai dogs are less trained or less used to be left alone, possibly because it is usual for Thai households to have a housekeeper or maid, so that there is someone in the house most of the time [55]. Parthasarathy \& Crowell-Davis [56] stated that dogs with separation anxiety/separation-related problems may have a different type of attachment relationship with their owners, which makes it more difficult for the dogs to adapt to stressful situations. Mariti et al. [57] showed that not all dog owners are able to correctly address the stress signals of their dogs, which could lead to behaviour problems. Separation anxiety/separation-related behaviour can have different causes [58]. For instance, it is possible that Thai dogs are allowed to roam free to a greater extent than Dutch dogs [18] and hence show greater anxiety/ separation-related problems when they are confined to the home alone. But these behaviour problems are not necessarily related to anxiety, and can also reflect relative inactivity [59]. Other QoL scales (social, environmental) were also influenced by culture. Overall, the BCS influenced the physical part of the QoL, and culture had a significant influence on the psychological, social and environmental scales of the QoL.

\section{Control over feeding and exercise}

The questionnaire of Rohlf et al. [6] is based on TPB. It was found that intentions to feed and exercise were best predicted by specific behavioural beliefs or control beliefs, but not by normative beliefs. However, in the original study, Rohlf et al. found only a small proportion of the BCS variance to be explained by feeding behaviours, intentions, and beliefs; none of the exercise variables explained the BCS [6]. We found that the attitude to exercise was an issue for Dutch dog owners and varied significantly between the different BCS groups. This was not the case for the Thai owners (Additional file 1: Table S6). 
As the BCS increased, the owners appeared to have less control over feeding and exercise. This is consistent with the findings of Kienzle et al. [10], who reported that the owners of obese dogs believed exercise to be relatively unimportant and that they lacked knowledge about feeding and exercise. In our study, the owners who indicated that they did not exercise their dog enough because they did not like exercising it, had dogs with a higher BCS. The owners of overweight/obese dogs weren't psychometrically tested of course, but it seems they are more self-centred and put greater emphasis on their own needs than did the owners of normal-weight dogs. Rohlf et al. [6] also found significant correlations between owners' behavioural beliefs and barriers, normative beliefs and perceptions of control and owners' behaviour regarding the feeding and exercise of their dogs. Our findings indicate that owner attitudes and beliefs essentially cause obesity because of lack of knowledge and perceived control. This supports the contention of Kienzle et al. [10] that the owners of obese dogs find exercise less important than do the owners of normal weight dogs. Roudebush et al. [60] found that increased physical activity and environmental enrichment are beneficial to overweight dogs and their owners.

In Thailand, dog-centred owners may be influenced by Buddhist beliefs, by which giving food to a dog is good karma. There was a strong cultural influence on the subscale 'Feed to please' (Additional file 1: Table S6). Thai owners did this, as was expected, significantly more than did Dutch owners, yet this occurred more often in dogs with low BCS than in dogs with a high BCS, which was unexpected. Dutch dog owners also feed to please, as an expression of their love for their pet [61]. According to Kienzle et al. [10] "owners argue that they love their pets so much that they just can't deny it treats in the form of food". This belief contributes to overweight and obesity. Further, with a modern life-style, more self-centred owners may have, or take, less time to exercise their dogs [11].

The attitude in feeding of the Dutch dog owners significantly influenced their dog's BCS whereas this was not the case for the Thai owners (Fig. 3, panel A). This might be because Thai and Dutch owners have a different, culturally determined, bond with their dogs.

\section{Conclusions}

In this study, the Likert-scale questionnaires designed by Schneider et al. [12] and Rohlf et al. [6] were transformed into continuous scales (visual analogue scales), which enabled more powerful statistical techniques to be used and the calculation of integrated z-scores. The magnitude of the factor loadings was similar to that of Study 2 (ill dogs) of Schneider et al. [12]. On the basis of owner-reported information, the QoL of overweight and obese dogs was mainly influenced by the physical status of the dog, whereas psychological aspects of QoL were influenced more by culture
(Thai or Dutch) than by BCS. In the Netherlands, owner attitudes to feeding and exercise significantly influenced the BCS. As there were differences on these subscales between the two countries, dog owners in these two countries would appear to have significantly different attitudes towards feeding and exercise. The only exception was when owners seemed to be more self-centred and had less control over feeding, in which case the BCS was increased in dogs in both countries. Obesity in pets is influenced by owner attitudes regarding feeding and exercise, and it is very well possible that the same attitudes influence the health status of the owners. This could explain why there is a correlation between obesity in owners and their dogs [21]. Obesity in both humans and dogs involves a complex multiplicity of genetic and environmental factors [62]. The One Health approach can help to unravel these factors [22].

\section{Additional file}

Additional file 1: Table S1. Owner demographics and dog food information: categorical data, Table S2 Dog activities: continuous data, Table S3. Orthogonal factor loadings per scale for owner-reported quality of life. Table S4. Orthogonal factor loadings per scale for owner attitude, Table S5. Owner-reported quality of life: subscale z-scores, Table S6. Owner attitude: subscale z-scores. Table S7. Meaningful comparisons of orthogonal factor loadings for quality of life from this study and the two studies of Schneider et al. [12]. Figure S1. Association between years of dog ownership and dog age. Panel A, Thailand; panel B, the

Netherlands. Figure S2. Association between body condition score and dog age. Appendix. the questionnaire. (DOCX $283 \mathrm{~kb}$ )

\begin{abstract}
Abbreviations
ANCOVA: Analysis of covariance; ANOVA: Analysis of variance; BCS: Body condition score; KU-VTH: Kasetsart University Veterinary Teaching Hospital; PAA: Principal axis analysis; PCA: Principal component analysis; QoL: Quality of Life; SD: Standard deviation; SEM: Standard error of the mean ; TPB: Theory of planned behaviour
\end{abstract}

\section{Acknowledgments}

The authors are grateful to E.M. Smulders and B.D. van der Baan for the Dutch data collection. We thank J. Sykes for checking the English and we also warmly thank M. Labots for preparing Figs. 2 and 3. The authors are also grateful to the two anonymous reviewers, an associate editor and the editor for their valuable comments on the manuscript, which has undoubtedly helped the authors to improve the article. This article is a tribute to the memory of Prof. dr. Frauke Ohl who passed away on 28 January 2016. We remember Frauke as a dedicated scientist and an inspiring and powerful boss, colleague and friend.

Availability of data and materials

All the data (including Additional files) supporting our findings are contained within this article. Raw data involved in the current study are available on request to the corresponding author. All materials are described within this article.

\section{Authors' contributions}

Study conception and design: NE and HAvL, Acquisition of data: SS and CC, Analysis and interpretation of data: HAVL, SS and NE, Drafting of manuscript: NE and HAvL, Critical revision: NE and HAvL. All authors read and approved the final manuscript

\section{Ethics approval and consent to participate}

Ethical approval for this study was granted by the Faculty of Veterinary Medicine from both Utrecht University (the Netherlands) and Kasetsart University (Thailand). This study did not include animal experiments. 


\section{Competing interests}

The authors declare that they have no competing interests.

\section{Publisher's Note}

Springer Nature remains neutral with regard to jurisdictional claims in published maps and institutional affiliations.

\section{Author details}

'Division Human-Animal Relations, Department of Animals in Science and Society, and Animal Behaviour Clinic, Faculty of Veterinary Medicine, Utrecht University, Yalelaan 2, PO Box 80166, 3508, TD, Utrecht, The Netherlands. ${ }^{2}$ Division of Animal Welfare \& Laboratory Animal Science, Department of Animals in Science and Society, Faculty of Veterinary Medicine, Utrecht University, Utrecht, The Netherlands. ${ }^{3}$ Department of Companion Animal Clinical Sciences, Faculty of Veterinary Medicine, Kasetsart University, Bangkok, Thailand. ${ }^{4}$ Presenr address: Department of Clinical Sciences, College of Veterinary Medicine \& Biomedical Sciences, Colorado State University, Denver, USA. ${ }^{5}$ Brain Center Rudolf Magnus, University Medical Center Utrecht, Utrecht, The Netherlands.

\section{Received: 8 April 2016 Accepted: 19 June 2018}

\section{Published online: 09 July 2018}

\section{References}

1. Vis B, Hylands T. Fat government, thin populace? Is the growth of obesity prevalence lower in more generous welfare states? Int I Soc Welfare. 2013; 22(4):360-73.

2. German AJ. The growing problem of obesity in dogs and cats. J Nutr. 2006; 136(7 Suppl):1940S-6S

3. Gosselin J, Wren JA, Sunderland SJ. Canine obesity- an overview. J Vet Pharmacol Ther. 2007;30(Suppl 1):1-10.

4. Becker N, Dillitzer N, Sauter-Louis C, Kienzle E. Feeding of dogs and cats in Germany. Tierärtzl Prax Kleintiere. 2012;2012(6):391-7.

5. Courcier EA, Mellor DJ, Thomson RM, Yam PS. A cross sectional study of the prevalence and risk factors for owner misperceptions of canine body shape in first opinion practice in Glasgow. Prev Vet Med. 2011;102(1):66-74.

6. Rohlf VI, Toukhsati S, Coleman GJ, Bennett PC. Dog obesity: Can dog caregivers'(owners') feeding and exercise intentions and behaviors be predicted from attitudes? J Appl Anim Welf Sci. 2010;13(3):213-36.

7. Jeusette I, Detilleux J, Cuvelier C, Istasse L, Diez M. Ad libitum feeding following ovariectomy in female Beagle dogs: Effect on maintenance energy requirement and on blood metabolites. J Anim Physiol Anim Nutr. 2004;88(3-4):117-21.

8. Laflamme P. Nutrition for aging cats and dogs and the importance of body condition. Vet Clin N Am -Small Anim Pract. 2005;35(3):713-42.

9. McGreevy PD, Thomson PC, Pride C, Fawcett A, Grassi T, Jones B. Prevalence of obesity in dogs examined by Australian veterinary practices and the risk factors involved. Vet Rec. 2005;156(22):695-702.

10. Kienzle E, Bergler R, Mandernach A. Comparison of the feeding behaviour of the man-animal relationship in owners of normal and obese dogs. J Nutr. 1998;128(12):2777S-82S.

11. Robertson ID. The association of exercise, diet and other factors ownerperceived obesity in privately owned dogs from metropolitan Perth WA. Prev Vet Med. 2003;58(1-2):75-83.

12. Schneider TR, Lyons JB, Tetrick MA, Accortt EE. Multidimensional quality of life and human-animal bond measures for companion dogs. J Vet Behav Clin Appl Res. 2010;5(6):287-301.

13. Klinkenberg $\mathrm{H}$, Sallander MH, Hedhammer A. Feeding, exercise and weight identified as a risk factor in Canine Diabetes Mellitus. J Nutr. 2006:136(7 Suppl):1985S-7S.

14. Bell D, Galloway S, Hamilton C, Scullion S. Quality of life and well-being: Measuring the benefits of culture and sport: Literature review and thinkpiece. 2006. Scottish Executive Social Research; http://www.gov.scot/ resource/doc/89281/0021350.pdf

15. Marinelli L, Adamelli S, Normando S, Bono G. Quality of life of the pet dog: Influence of owner and dog's characteristics. Appl Anim Behav Sci. 2007; 108(1-2):143-56

16. Brown PJ. Culture and the evolution of obesity. Hum Nat. 1991:2(1):31-57.

17. Hawks SR. Human culture and the global epidemic of obesity. Health Weight J. 2001:15(6):85-8.

18. Chunekamrai, S. (2015) Personal communication.
19. Burkholder WJ, Toll PW. Obesity. In: Hand MS, Thatcher CD, Reimillard RL, Roudebush P, Morris ML, Novotny BJ, editors. Small Animal Clinical Nutrition. 4th ed. Topeka: Mark Morris Institute. p. 401-30.

20. Bland IM, Guthrie-Jones A, Taylor RD, Hill J. Dog obesity: owner attitudes and behavior. Prev Vet Med. 2009;92(4):333-40.

21. Nijland ML, Stam F, Seidell JC. Overweight in dogs, but not in cats, is related to overweight in their owners. Public Health Nutr. 2010;13(1):102-6.

22. Sandøe P, Palmer C, Corr S, Astrup A, Bjornvad CR. Canine and feline obesity: a One Health perspective. Vet Rec. 2014;175(24):610-6.

23. Day MJ. One Health: the small animal dimension. Vet Rec. 2010;167(22):847-9.

24. Ajzen I. The theory of planned behavior. Organ Behav Hum Perform. 1991; 50(2):179-211.

25. Coleman GJ, McGregor M, Hemsworth PH, Boyce J, Dowling S. The relationship between beliefs, attitudes and observed behaviors of abattoir personnel in the pig industry. Appl Anim Behav Sci. 2003:82(3):189-200.

26. Godin G, Kok G. The theory of planned behavior : A review of its applications to health-related behaviors. Am J Health Promot. 1996;11(2):87-98.

27. Zwart H. A short history of food ethics. J Agric Environ Ethics. 2000;12(2):113-26.

28. Phelps N. The Great Compassion: Buddhism and Animal Rights. Herndon: Lantern Books; 2004.

29. Wojciechowska JL, Hewson CJ. Quality-of-life assessment in pet dogs. J Am Vet Med Assoc. 2005;226(5):722-8.

30. Wiseman-Orr ML, Scott EM, Reid J, Nolan AM. Validation of a structured questionnaire as an instrument to measure chronic pain in dogs on the basis of effects on health-related quality of life. Am J Vet Res. 2006;67(11):1826-36.

31. Yeates JW, Main D. Assessment of companion animal quality of life in veterinary practice and research. J Small Anim Pract. 2009;50(6):274-81.

32. Krumpal I. Determinants of social desirability bias in sensitive surveys: a literature review. Qual Quant. 2013;47(4):2025-47.

33. Chen $\mathrm{H}-\mathrm{Y}$, Boore JRP. Translation and back-translation in qualitative nursing research: methodological review. J Clin Nurs. 2009;19:234-9.

34. Schellingerhout JM, Heymans MW, Verhagen AP, de Vet HC, Koes BW, Terwee CB. Measurement properties of translated versions of neck-specific questionnaires: a systematic review. BMC Med Res Methodol. 2011;11:87.

35. Conover WJ, Iman RL. Rank transformations as a bridge between parametric and nonparametric statistics. Am Stat. 1981;35(3):124-9.

36. Scheirer J, Ray WS, Hare N. The analysis of Ranked data derived from completely randomized factorial designs. Biometrics. 1976:32(2):429-34.

37. Budaev SV. Using principal components and factor analysis in animal behavior research: caveats and guidelines. Ethology. 2010;116(5):472-80.

38. Stafford K. The Welfare of dogs. Volume 4 of the series Animal Welfare. The Netherlands: Springer, Dordrecht; 2007.

39. Witzel AL, Kirk CA, Henry GA, Toll PW, Breida JJ, Paetau-Robinson I. Use of a novel morphometric method and body fat index system for estimation of body composition in overweight and obese dogs. J Am Vet Med Assoc. 2014;244(11):1279-84.

40. German AJ, Holden SL, Moxham GL, Holmes KL, Hackett RM, Rawlings JM. A simple, reliable tool for owners to assess the body condition of their dog or cat. J Nutr. 2006:136(7 Suppl):2031S-3S.

41. Larsen JA, Villaverde C. Scope of the problem and perception by owners and veterinarians. Vet Clin N Am - Small Anim Pract. 2016:46(5):761-72.

42. Schauf S, Salas-Mani A, Torre C, Bosch G, Swarts H, Castrillo C. Effects of sterilization and of dietary fat and carbohydrate content on food intake, activity level, and blood satiety-related hormones in female dogs. J Anim Sci. 2016:94(10):4239-50.

43. Dean RS. The use and abuse of questionnaires in veterinary medicine. Equine Vet J. 2015:47(4):379-80

44. Brislin RW. Back-translation for cross-cultural research. J. Cross-Cult Psychol. 1970;1(3):185-216.

45. Tuleja EA, Beamer L, Shum C, Chan EKY. Designing and developing questionnaires for translation tutorial. IEEE Trans Prof Commun. 2011;54(4):392-405.

46. Lange F, Söderlund M. Response formats in questionnaires: itemized rating scales versus continuous rating scales. SSE Working Paper Series in Business Administration. 2004:13:1-16.

47. Treiblmaier $\mathrm{H}$, Filzmoser $\mathrm{P}$. Benefits from using continuous rating scales in online surevey research. Forschungsbericht Institut für Statistik und Wahrscheinlichkeitstheorie, Technische Universität Wien. 2009;SM-2009-4:1-29.

48. Rusch T, Lowry PB, Mair P. Treiblmaier H. Breaking free from the limitations of classical test theory: Developing and measuring information systems scales using item response theory. Inf Manage. 2016;doi:https://doi.org/10 1016/j.im.2016.06.005. 
49. McKelvie SJ. Graphic rating scales - How many categories. Br J Psychol. 1978;69:185-202.

50. Hasson D, Arnetz BB. Validation and findings comparing VAS vs. Likert scales for psychosocial measurements. Int Elec. J Health Educ. 2005;8:178-92.

51. Field A. Discovering Statistics Using SPSS. 3rd ed. London: SAGE Publication Ltd; 2009.

52. Pancharoen C, Thisyakorn U, Lawtongkum W, Wilde H. Rabies exposures in Thai children. Wildern Environ Med. 2001;12(4):239-43.

53. Lalwani AK, Shavitt S, Johnson T. What is the relation between cultural orientation and socially desirable responding? J Personal Soc Psychol. 2006; 90(1):165-78.

54. Mills $\mathrm{D}$, van der Zee $\mathrm{E}$, Zulch $\mathrm{H}$. When the bond goes wrong: problem behaviours in the social context. In: The Social Dog - Behaviour and Cognition, edited by Kaminksi J \& Marshall-Pescini S. 2014, Academic Press, pp. 223-45.

55. Willis KD, Yeoh BSA. Gender and transnational household strategies: Singaporean migration to China. Reg Stud. 2000;34(3):253-64.

56. Parthasarathy V, Crowell-Davis SL. Relationship between attachment to owners and separation anxiety in pet dogs (Canis lupus familiaris). J Vet Beh. 2006;1 (3):109-20.

57. Mariti C, Gazzano A, Landsdown Moore J, Baragli P, Chelli L, Sighiery C. Perception of dogs' stress by their owners. J Vet Behav - Clin Appl Res. 2012;7(4):213-9.

58. Appleby D, Pluijmakers J. Separation anxiety in dogs: The function of homeostasis in its development and treatment. Vet Clin N Am - Small Anim Pract. 2003;33(2):321-44.

59. Clubb R, Mason G. Captivity effects on wide-ranging carnivores. Nature. 2003;425(2):473-4.

60. Roudebush P, Schoenherr WD, Delany SJ. An evidence-based review of the use of therapeutic foods, owner education, exercise, and drugs for the management of obese and overweight pets. J Am Vet Med Assoc. 2008; 233(5):717-25.

61. Simons J. De invloed van obesitas op het welzijn van honden. Master thesis. 2012. (Utrecht University, the Netherlands).

62. Courcier EA, Thomson RM, Mellor DJ, Yam PS. An epidemiological study of environmental factors associated with canine obesity. J Small Anim Pract. 2010;51(7):362-7.

\section{Ready to submit your research? Choose BMC and benefit from:}

- fast, convenient online submission

- thorough peer review by experienced researchers in your field

- rapid publication on acceptance

- support for research data, including large and complex data types

- gold Open Access which fosters wider collaboration and increased citations

- maximum visibility for your research: over $100 \mathrm{M}$ website views per year

At BMC, research is always in progress.

Learn more biomedcentral.com/submissions 